\title{
A MULTI-PARAMETERS SPATIAL BIASING TECHNIQUE WITH MONTE CARLO METHOD USED IN SIMULATION OF NEUTRON TRANSPORT AND TRANSMISSION THROUGH PROTECTION SHIELDS
}

\author{
Abderrazak Khanouchi ${ }^{1 *}$, Lhoucine Oufni ${ }^{2}$, El Arbi Siher ${ }^{3}$ \\ and Abdellatif Jehouani ${ }^{4}$ \\ ${ }^{1}$ Faculty of Sciences and Technics BeniMellal, \\ Sultan Moulay Slimane University, Morroco \\ ${ }^{2}$ Faculty of Sciences and Technics BeniMellal, \\ Sultan Moulay Slimane University, Morroco \\ ${ }^{3}$ Faculty of Sciences and Technics BeniMellal, \\ Sultan Moulay Slimane University, Morroco \\ ${ }^{4}$ Faculty of Sciences Semlalia Marrakech, Cadi Ayyad University, Morroco \\ ${ }^{1}$ kabd70@gmail.com, ${ }^{2}$ 1.oufni@usms.ma, ${ }^{3}$ e.siher@usms.ma, \\ 4a.jehouani@ucam.ma
}

\begin{abstract}
Frequently, the neutron transmission through the shields used for protection against radiation is an unavoidable phenomenon, so we have interested in this work to study the neutron transmission through shields. We have considered an infinite homogenous slab witch characterized by his scattering probability noted Ps, with a different thickness and an infinite plane source of neutrons which arrived on the left side of the slab and on the right side detector with fixed window is placed to detect transmitted neutrons and evaluate the neutron transmission probabilities. We used the simulation Monte Carlo method for sampling the neutron history in the slab and in order to accelerate the calculation convergence we have developed a new multi-parameters spatial biasing technique with 4 parameters. For each thickness of the slab and for several values of Ps we have determined the detector response and calculated the neutron transmission probability. We compared our result by results obtained with the spatial biasing technique with 2 parameters and 1 parameter. Then we have determined the FOM (Figure of the Merit) for each method. We can also notice that our method presents bests results by obtaining the greatest FOM for a large thickness of the slab having high scattering probability Ps.
\end{abstract}

Keywords - Visualization, Simulation, Neutron, transmission, Protection Screen, Monte Carlo, Spatial Biasing, Multi-parameters

\section{INTRODUCTION}

The neutron transmission through shields used as protection screen against radiation is an unavoidable phenomenon $[1 ; 2]$. Then the protection against radiation constitutes one of important concerns of reactor physicists [3; 4], so that, in this work, we have interested to contribute in the amelioration of protection calculations. Then we consider an infinite 
plane source which produces a parallel beam of neutrons which arrived on the left side of an infinite homogeneous slab, with a variable thickness equal to $n \lambda$ (where $\lambda$ is the mean free path of the neutron in the slab and $n$ is a positive natural number). Neutrons will undergo collisions inside the slab and may be transmitted through the right boundary and detected by an infinite plane detector placed at the left side of the slab (Figure 1).

Our purpose is to evaluate the neutron transmission probability for neutrons transmitted through the slab and detected by the detector $[5 ; 6]$.

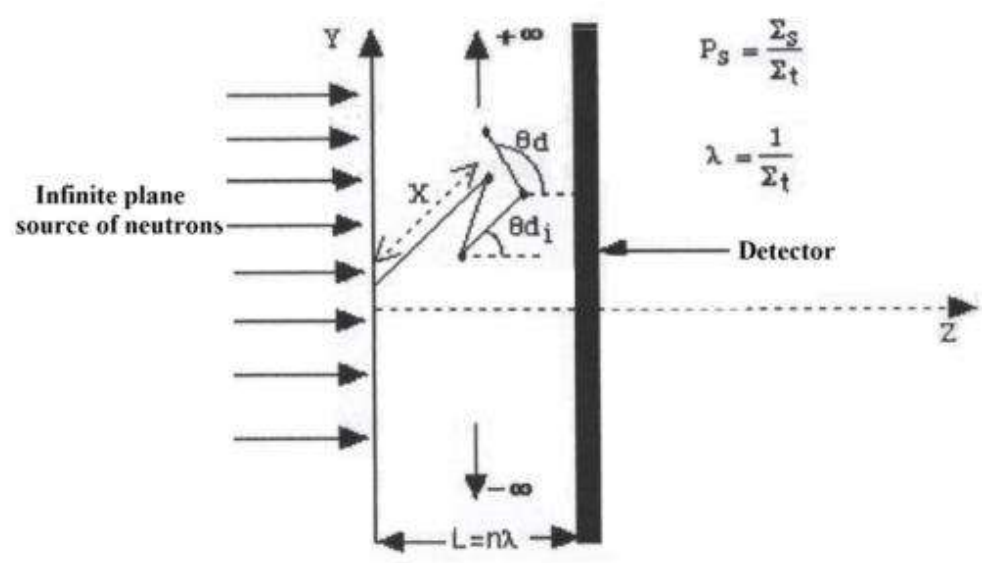

Fig. 1 Geometry of the problem used for neutron transmission study

\section{RESEARCH METHOD}

The Neutron history is generated by simulation with the Monte Carlo Method [7] and in order to accelerate the convergence of calculations [8] and make this method more efficient, by using a spatial biasing technique, we have used and compared three methods. All these three methods have recourse to the simulation with the Monte Carlo Method for sampling the different Neutron parameters such as its collision point, direction and path after collision, and by application of a spatial biasing technique:

- With one parameter for the first method [9] developed by Leo Levitt (BS1P)

- With two parameters for the second method [10; 11] developed by J Ghassoun (BS2P)

- With four parameters for the third method which we have developed and proposed in this work (BS4P)

The neutron parameters are generated by using probability densities of these sizes characterizing the neutron. Then the diffusion angle (scattering angle) after the collision is obtained by:

$$
\frac{\theta=\pi * \xi}{0 \leq \xi \leq 1}
$$

Where $\xi$ is a random number between 0 and 1

And we can determine the angle $\theta \mathrm{d}$ between the direction of neutron and the axis $\mathrm{Z}$ (figure 1) by:

$\theta d=\theta d i+\theta$ or $\theta d=\theta d i-\theta$

This is to take account if the diffusion is up or down the direction before the collision.

The neutron path after collision noted $\mathrm{X}$ is sampled by its natural probability density: 


$$
P(X)=\Sigma t * e^{-2 t * X}
$$

Where $\Sigma \mathrm{t}$ is the neutron total cross section. Then we can deduce:

$$
x=-\frac{\log (\xi)}{\Sigma t}
$$

Then, we can deduce:

$$
\boldsymbol{X}=-\log (\xi) * \lambda
$$

Then the neutron path is expressed in unit of $\lambda$.

In order to calculate the neutron transmission probability through the slab, we used the statistical weight method which consists to suppose that all neutrons are born at the source with a statistical weight equal to unit, and after each collision this statistical weight is multiplied by a factor Ps (scattering probability):

$$
P s=\frac{\Sigma s}{\Sigma t}
$$

With $\Sigma \mathrm{t}$ and $\Sigma$ s are respectively the total and scattering cross sections of the neutron to take accounts of the absorption. All transmitted neutrons contribute to results with a statistical weight between 0 and 1 . Then the neutron transmission probability noted $\mathrm{Pt}$ is defined as follows:

$$
P t=\frac{\sum_{\bar{i}=1}^{j s} W i}{N s}
$$

With: Wi is the neutron statistical weight and Ns is the neutron simulation number (neutron histories number).

The application of a spatial biasing technique consists to sample the neutron path after collision by the biasing probability density $\mathbf{P} *(\mathbf{X})$ :

$$
P^{*}(X) d X=\Sigma t^{*} * e^{-\Sigma t^{*} * X} d X \text { with } \Sigma t^{*}=a * \Sigma t
$$

Where $\Sigma \mathrm{t}$ and $\Sigma \mathrm{t}^{*}$ are respectively the natural and biasing total cross sections and a is a spatial biasing parameter.

For the first method using one parameter a we choose it between 0 and 1 and we can also note that $\Sigma t^{*}$ is less than $\Sigma t$ then the biasing path $\mathrm{X}^{*}$ is better than the natural path $\mathrm{X}$ :

$$
x^{*}=\frac{x}{a}
$$

It's the elongation of the path.

For others methods using multi-parameters we replaced the parameter a by four parameters a1, a2, a3 and a4 with:

$$
a \tilde{i}=1-b i * \mu \quad \text { where } \quad \mu=\cos \theta d
$$

Where $\boldsymbol{b} \boldsymbol{i}$ is a positive relative number.

After each collision we proceed to the correction of the neutron statistical weight as follows:

$$
W_{i}=W_{i-1} * P s * f c \quad \text { where } \quad f c=\frac{e^{-[1-a] 2 r e x}}{a}
$$


For the optimization of the parameter a, we used the equation proposed by Levitt as follows:

$$
a=P s^{n / 30} * e^{(1-a)}
$$

Then we noted that this parameter a depends on the thickness of the slab $\mathbf{n}$ and on the value of Ps.

For the second method BS2P we used a spatial biasing technique with 2 parameters a1 et a2 with $0<\mathrm{a} 1<\mathrm{a} 2<1$ when the neutron points to the detector $(\mu>0)$, this technique aims to lengthen the neutron path, but when the neutron come back to the left side $(\mu \leq 0)$ we don't used a spatial biasing technique but we used only the natural transformation.

For our method BS4P we developed a transformation with 4 parameters. When $(\mu>0)$ we apply the method BS2P but when the neutron come back to the left side $(\mu \leq 0)$ we apply another spatial biasing technique with 2 others parameters a3 and a4 (a4>a3>1). The purpose of this technique is to shorten and minimized the neutron path.

These parameters of biasing technique are given in the Tables 1,2 and shown in the Figures 2, 3.

Table I. Spatial biasing parameters case of the slab with thickness $40 \lambda$

\begin{tabular}{|c|c|c|c|c|c|}
\hline Ps & Parameter a & Parameter al & Parameter a2 & Parameter a3 & Parameter a4 \\
\hline 0,1 & 0,01737 & 0,00544 & 0,01 & 3 & 5 \\
\hline 0,2 & 0,04501 & 0,01186 & 0,03 & 2 & 6 \\
\hline 0,3 & 0,08003 & 0,019 & 0,077 & 2,5 & 9 \\
\hline 0,4 & 0,12256 & 0,0253 & 0,12 & 2,9 & 7 \\
\hline 0,5 & 0,17368 & 0,036 & 0,131 & 3 & 6 \\
\hline 0,6 & 0,23564 & 0,046 & 0,22 & 5,37 & 5,5 \\
\hline 0,7 & 0,31254 & 0,057 & 0,289 & 2,8 & 8 \\
\hline 0,8 & 0,41285 & 0,0687 & 0,41 & 1,95 & 4,1 \\
\hline 0,9 & 0,55916 & 0,081 & 0,562 & 5,6 & 20 \\
\hline
\end{tabular}

Table II. Spatial biasing parameters case of the slab with thickness $10 \lambda$

\begin{tabular}{|c|c|c|c|c|c|}
\hline Ps & Parameter a & Parameter a1 & Parameter a2 & Parameter a3 & Parameter a4 \\
\hline 0,1 & 0,21083 & 0,00544 & 0,07 & 10 & 50 \\
\hline 0,2 & 0,2865 & 0,0118 & 0,08 & 11 & 1000 \\
\hline 0,3 & 0,34919 & 0,02 & 0,09 & 22 & 500 \\
\hline 0,4 & 0,40735 & 0,0273 & 0,103 & 5 & 100 \\
\hline 0,5 & 0,46471 & 0,036 & 0,11 & 6 & 10 \\
\hline 0,6 & 0,52398 & 0,046 & 0,14 & 4 & 8 \\
\hline 0,7 & 0,58819 & 0,057 & 0,16 & 2 & 5 \\
\hline 0,8 & 0,66221 & 0,0687 & 0,20 & 4 & 8 \\
\hline 0,9 & 0,75784 & 0,081 & 0,23 & 5 & 10 \\
\hline
\end{tabular}




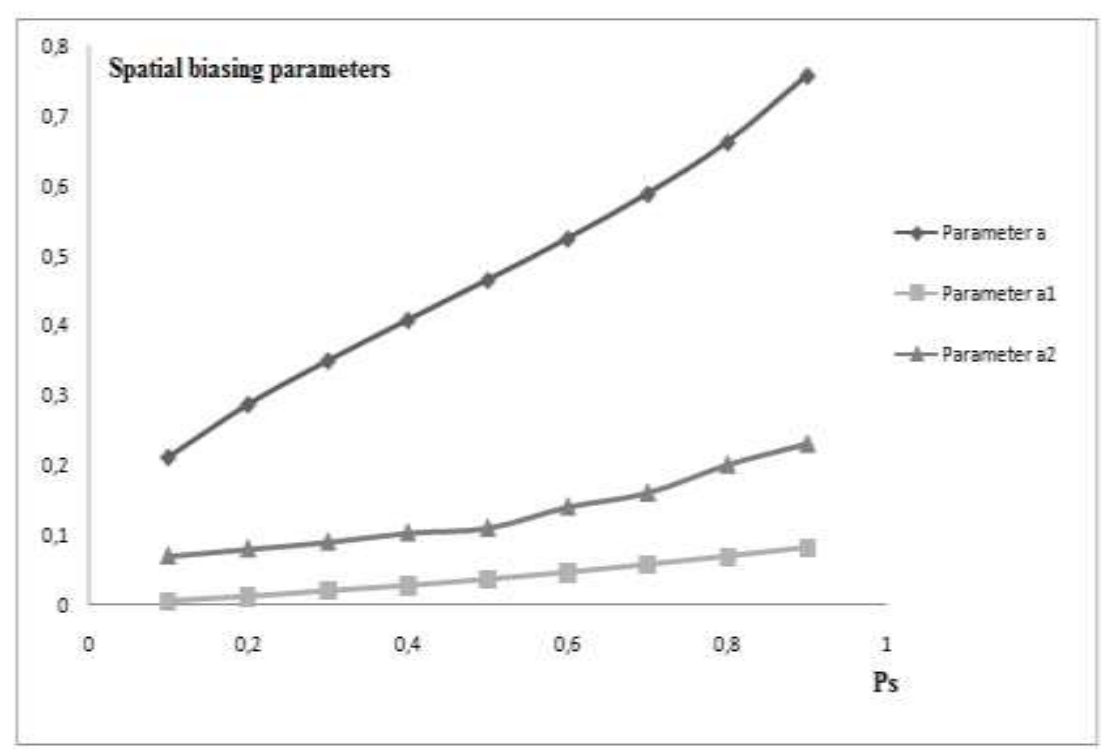

Fig. 2 Variation of spatial biasing parameters versus Ps case of the slab with thickness $10 \lambda$

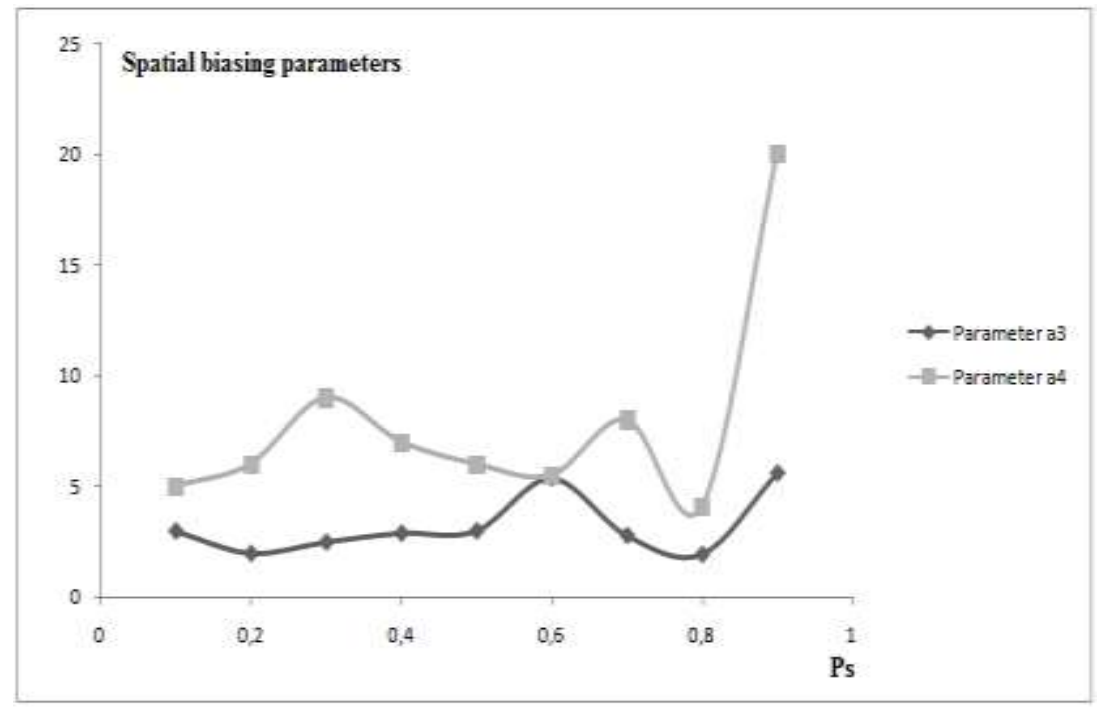

Fig. 3 Variation of spatial biasing parameters versus Ps case of the slab with thickness $40 \lambda$

\section{RESULTS AND ANALYSIS}

For several values of Ps and the thickness of the slab, we have used these three methods and calculated the neutron transmission probabilities. We presented results obtained at Figures 4 and 5. 


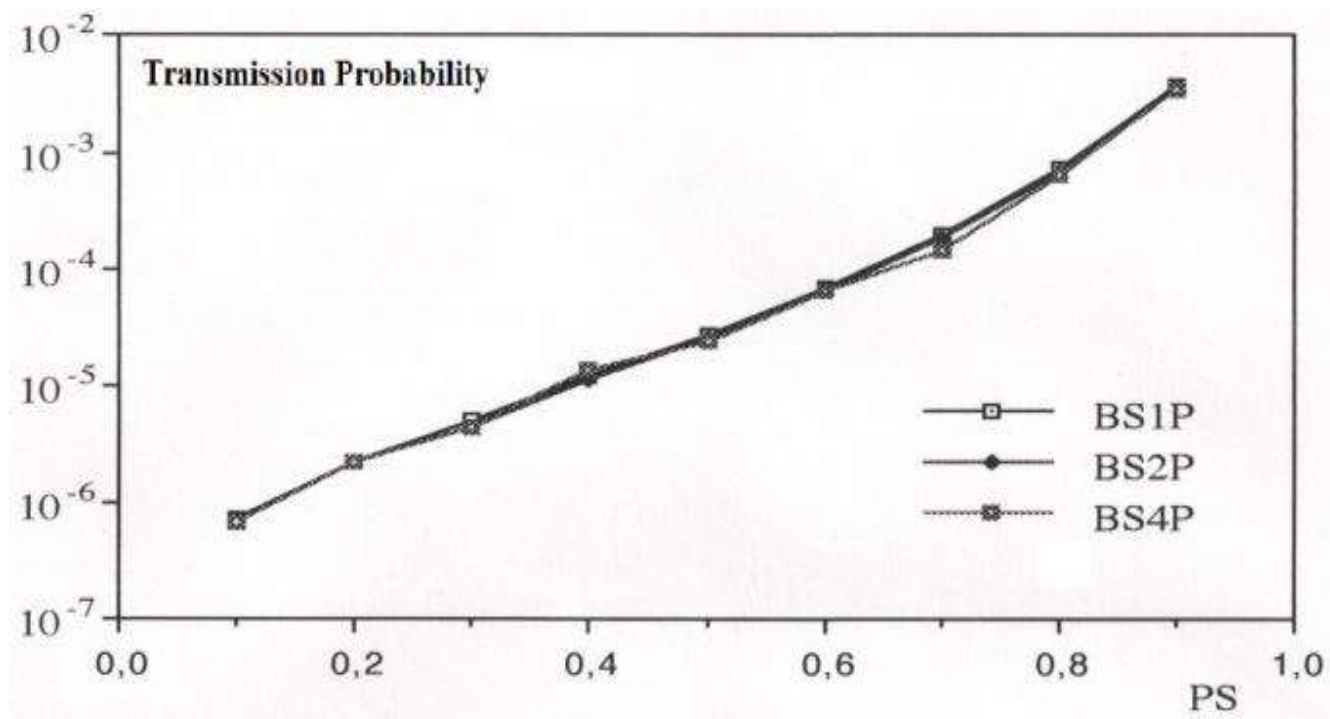

Fig. 4 Variation of transmission probability versus Ps case of the slab with thickness $10 \lambda$

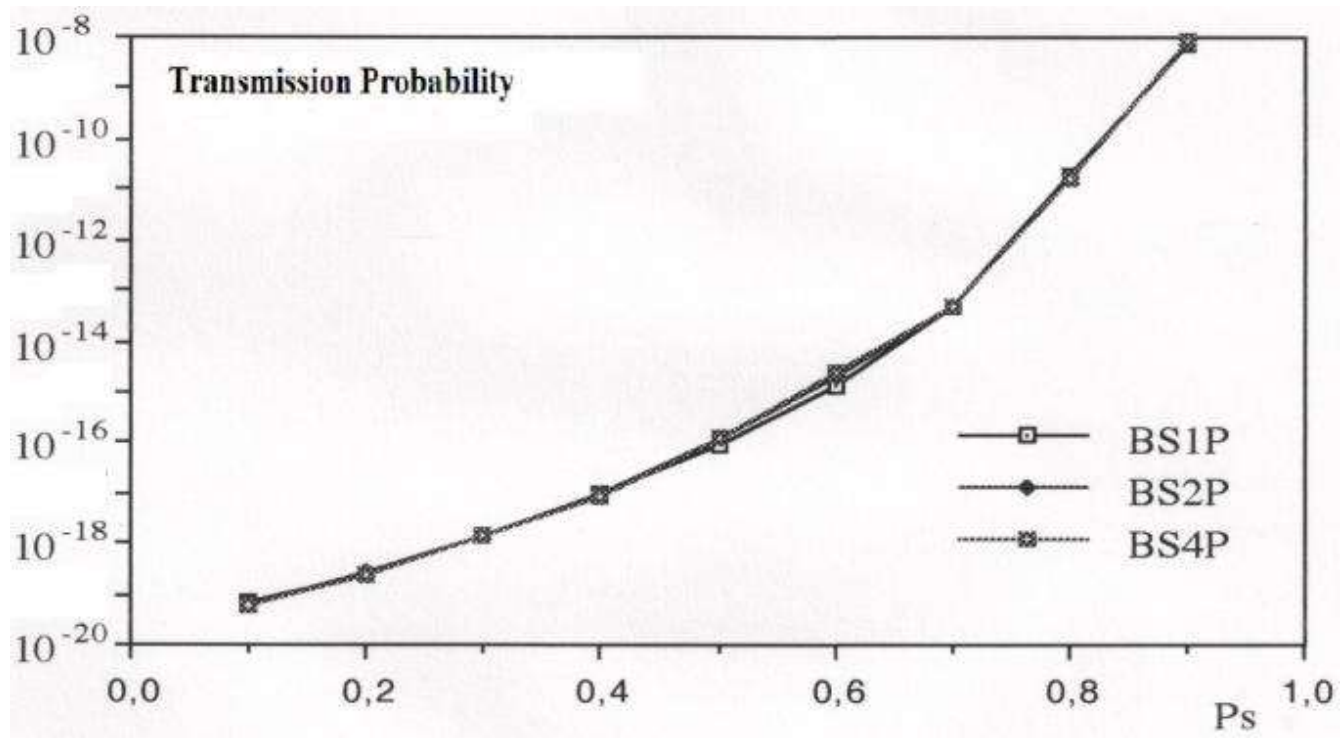

Fig. 5 Variation of transmission probability versus Ps case of the slab with thickness $40 \lambda$

We can also notice that the results obtained by these methods are in good agreement.

Now we discuss and compare some advantages of the three methods. Then we have compared the mean collision number obtained by these methods and we have calculated some Figures Of Merit noted FOM. This size involves the variance and the computer calculation time T:

$$
\text { FOM }=\frac{1}{T_{x} \sigma^{2}}
$$

So we notice that as FOM increases the method becomes more efficient.

The results obtained are presented in figures 6 to 11 . 


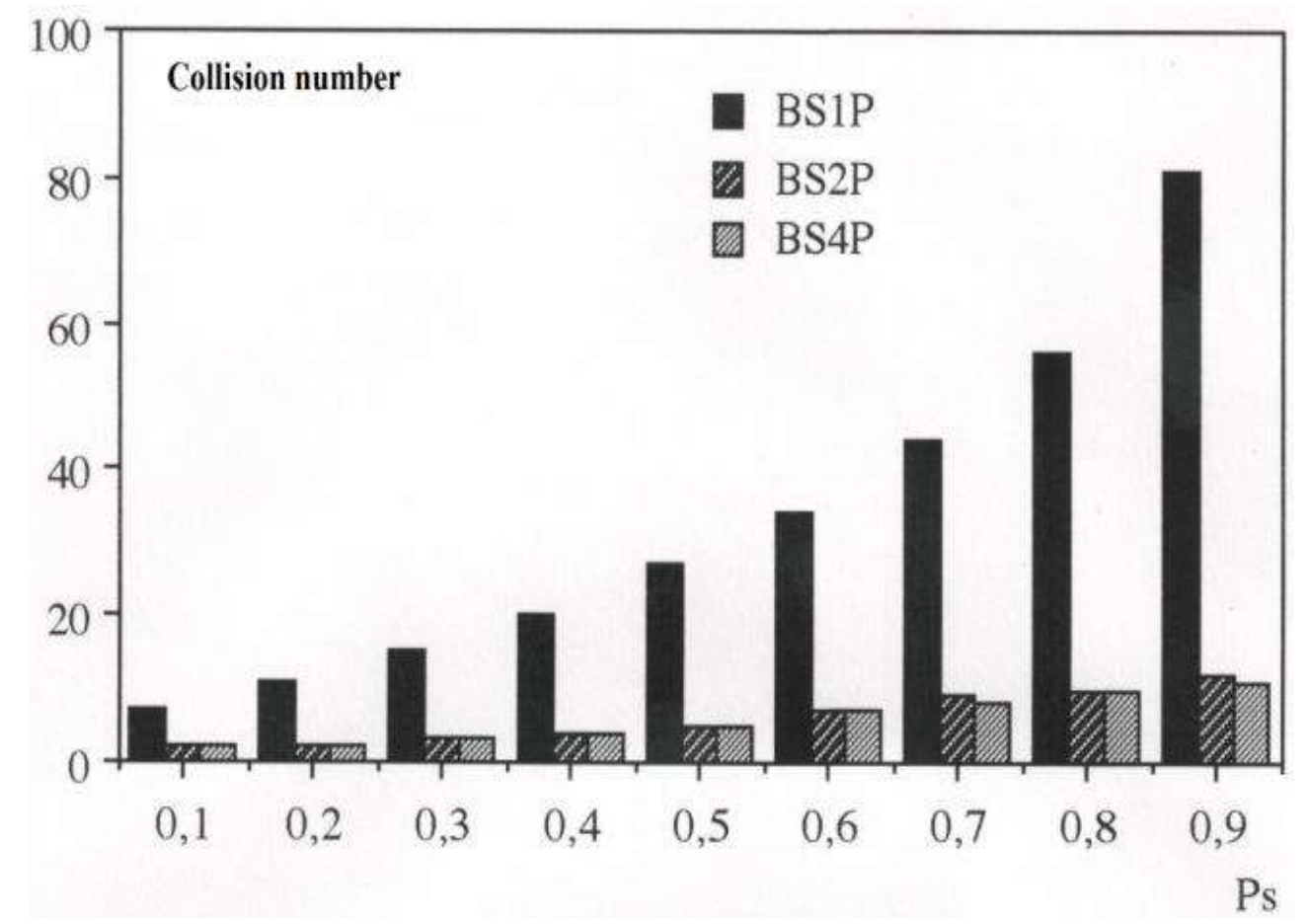

Fig. 6 Variation of the mean collision number versus Ps case of the slab with thickness $20 \lambda$

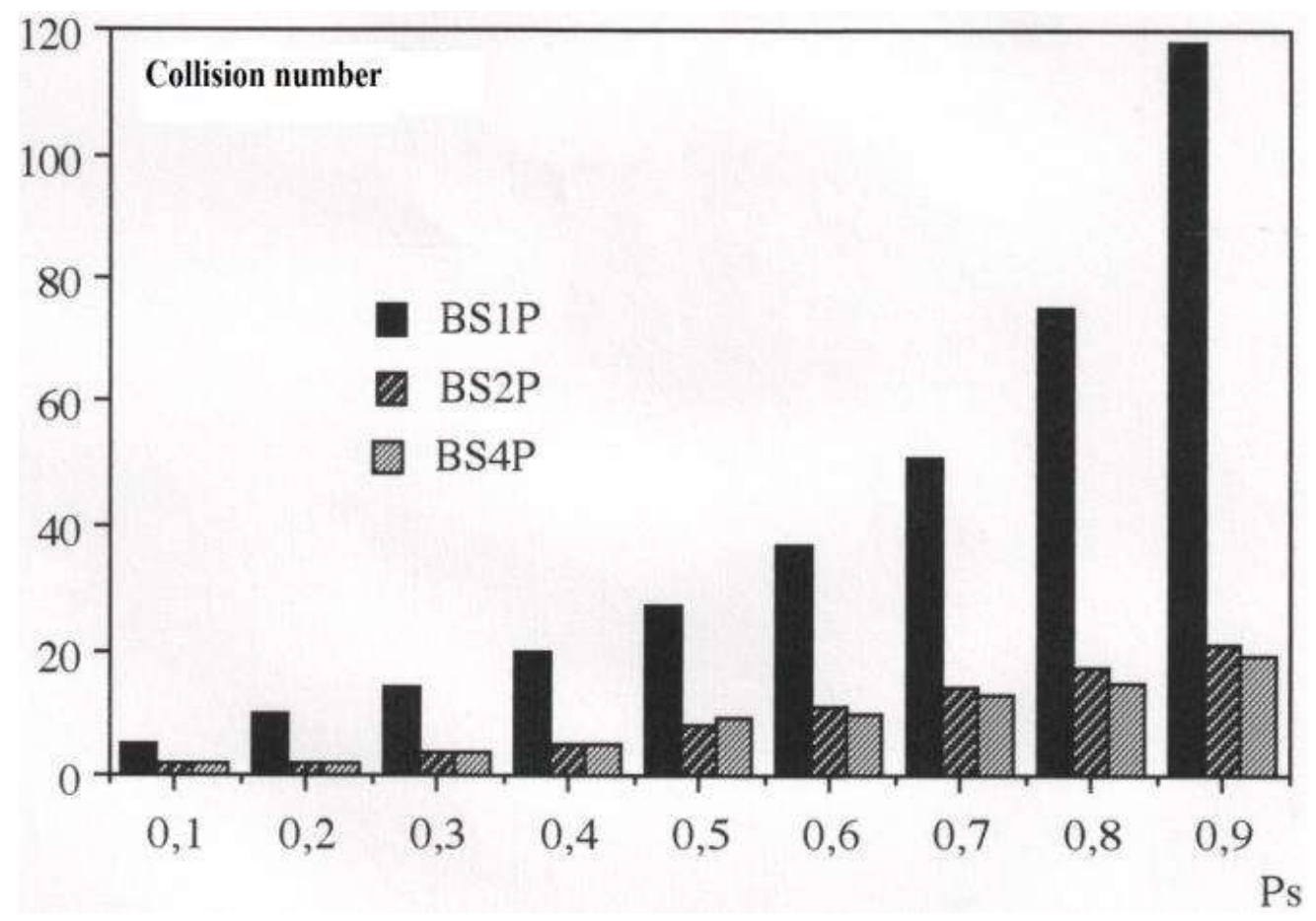

Fig. 7 Variation of the mean collision number versus Ps case of the slab with thickness $30 \lambda$ 


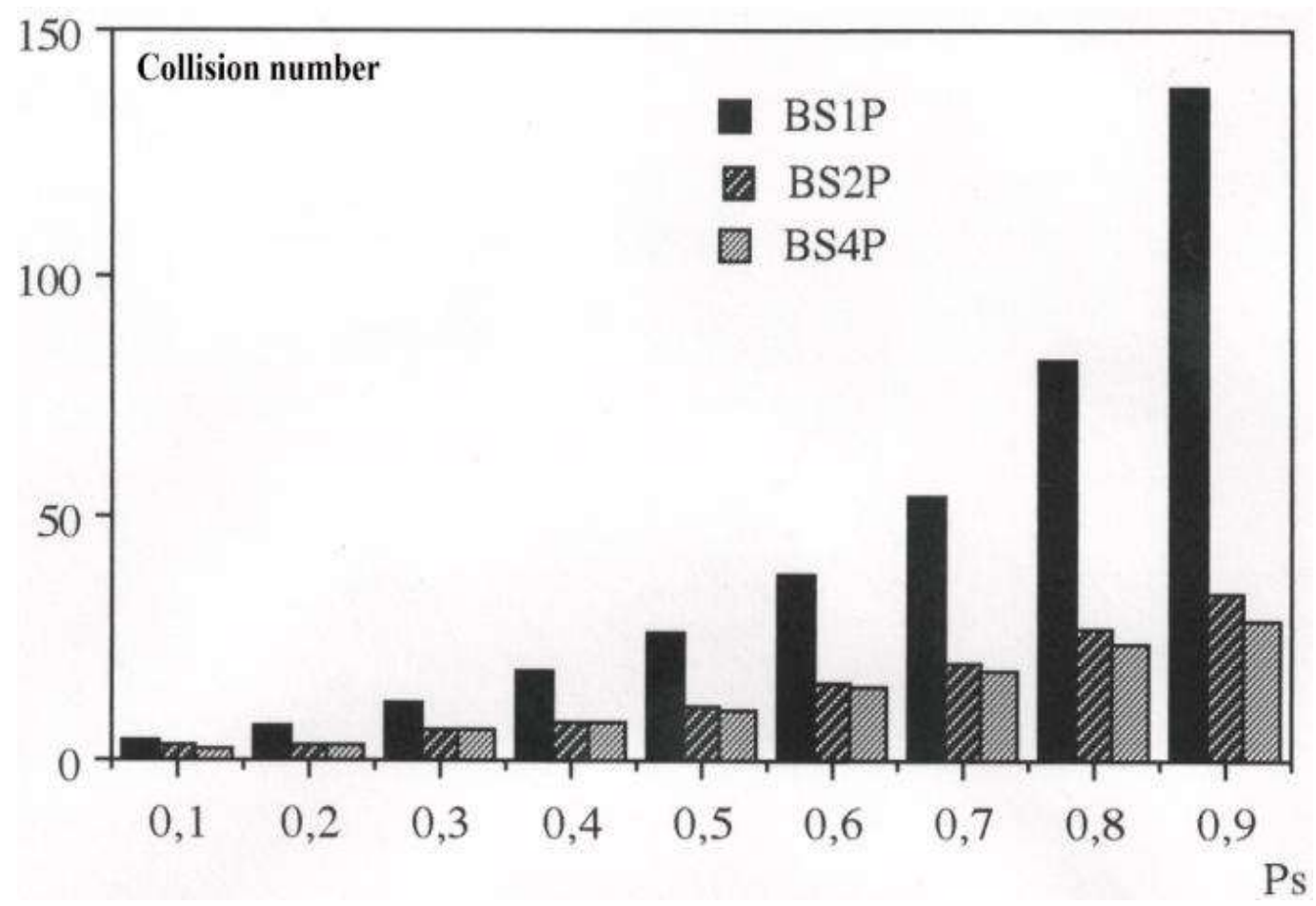

Fig. 8 Variation of the mean collision number versus Ps case of the slab with thickness $40 \lambda$

We note that the two methods BS4P and BS2P present a number of collisions significantly less than that obtained by the method BS1P, and therefore the neutrons spend less time to cross the slab, then these two methods have a gain in computation time.

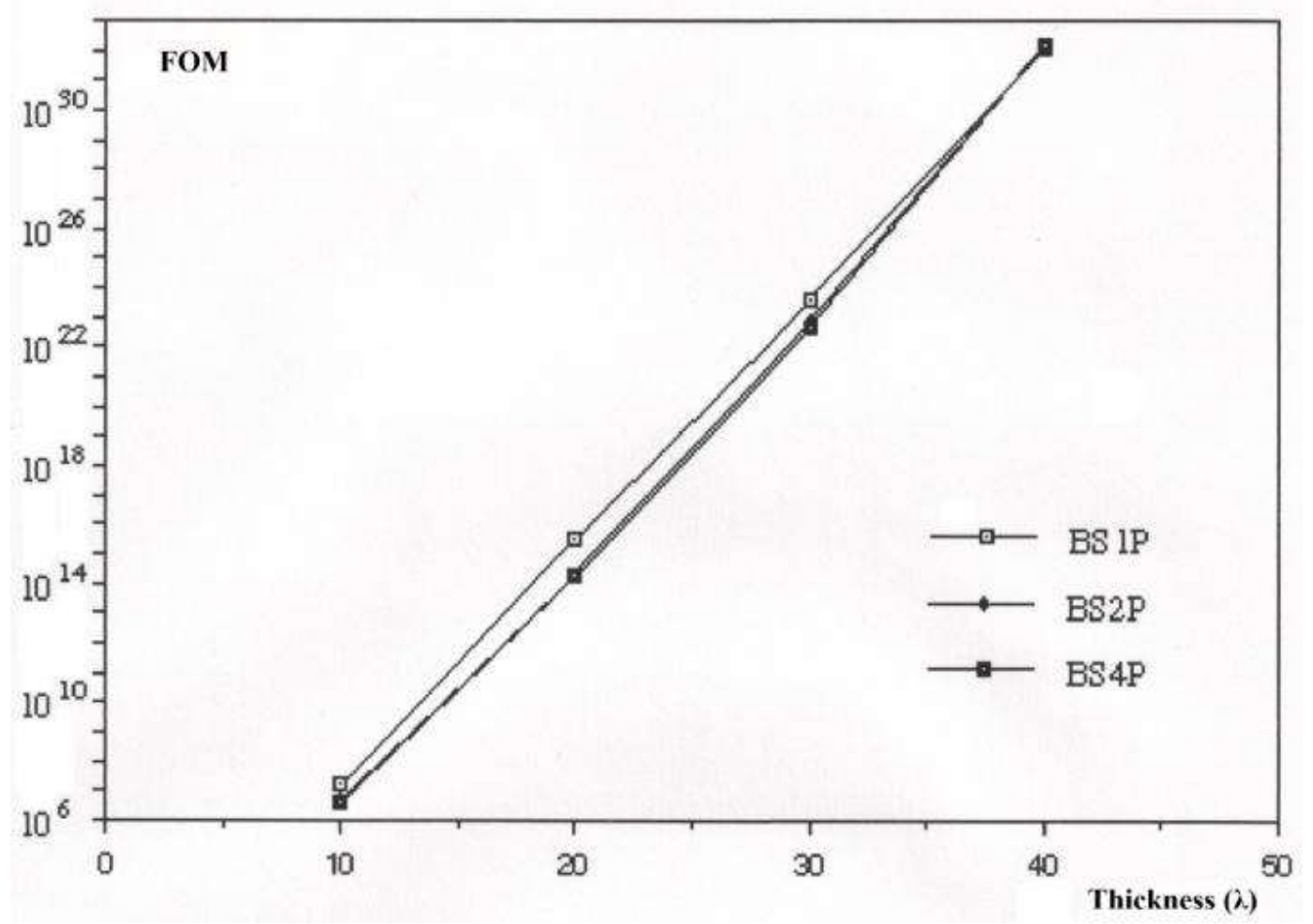

Fig. 9 Variation of FOM versus thickness case Ps=0.2 


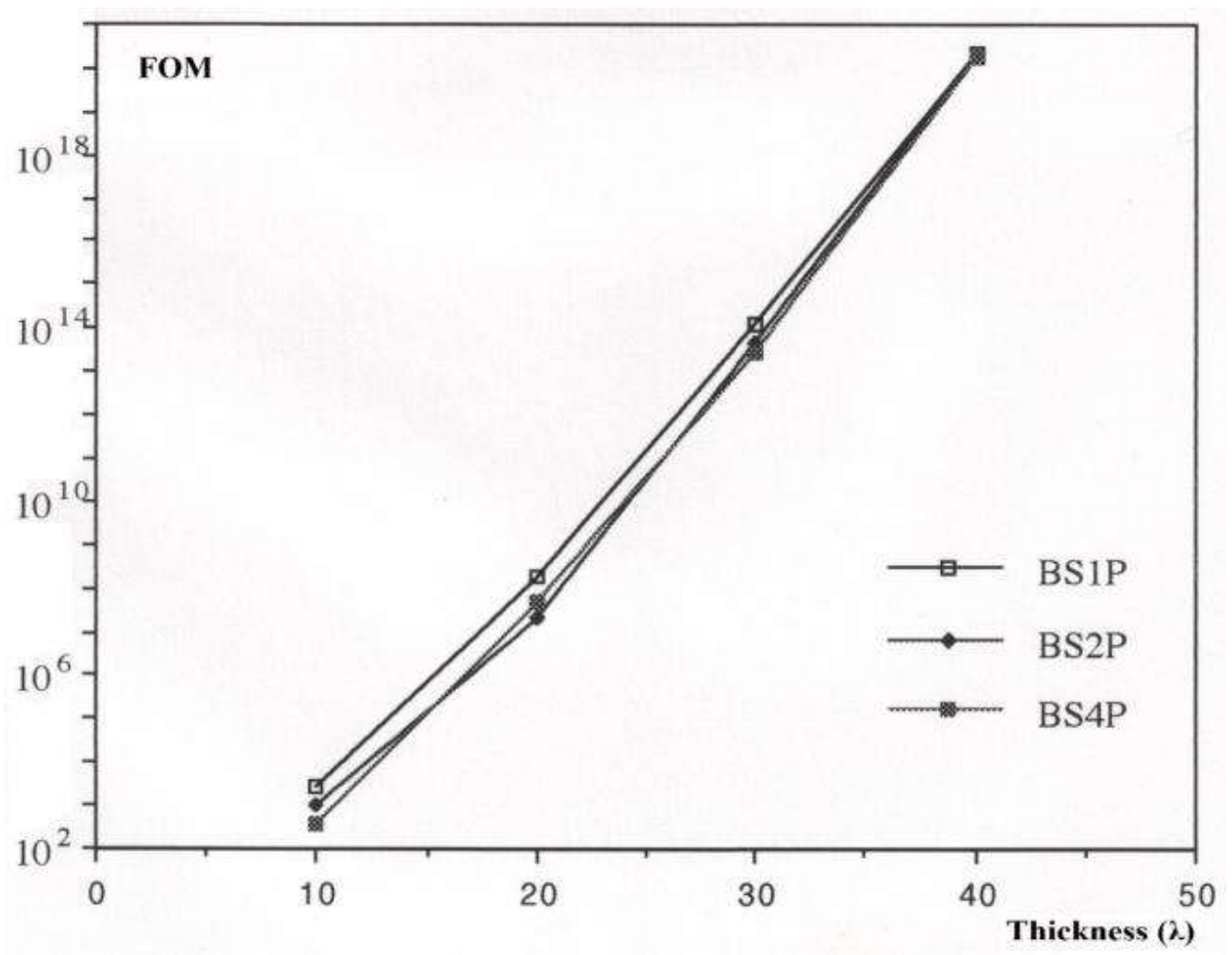

Fig. 10 Variation of FOM versus thickness case Ps=0.7

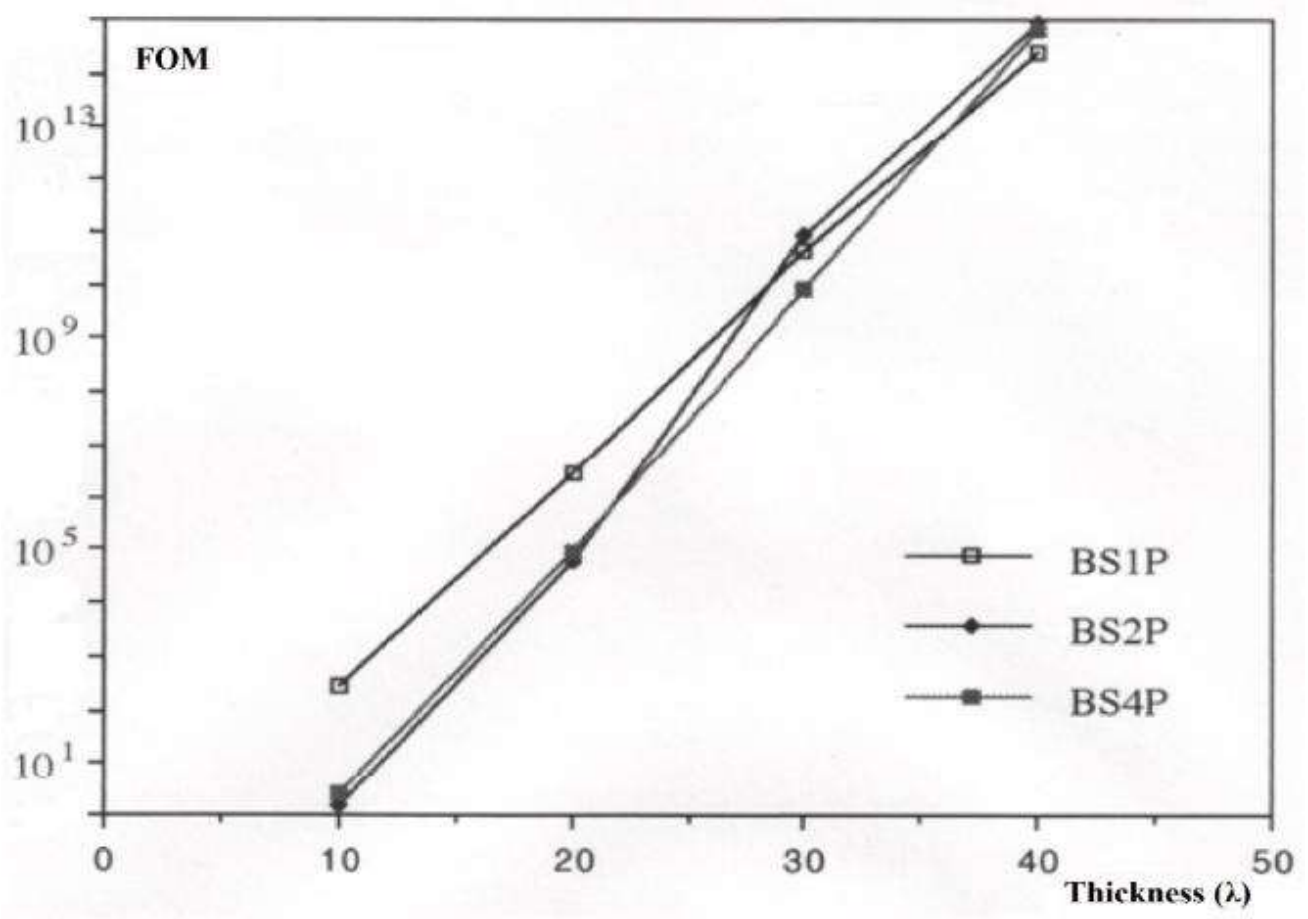

Fig. 11 Variation of FOM versus thickness case Ps $=0.8$

We can also see that our method BS4P presents best results for high Ps $(\mathrm{Ps}=0.8)$ and great values of thickness $(\mathrm{L}=40 \lambda)$.

The multi-parameters spatial biasing technique we developed in our study can be used by specialist practitioners in protection calculations to improve these 
calculations. Also this technique is a contribution that can allow the designer to make the optimal choice of protection conditions and desired and suitable choice of the material used in protection against radiations such as thick protection materials used in nuclear centers

\section{Conclusions}

In this work, we have developed a multi-parameters spatial biasing technique in order to make the Monte Carlo Method more efficient and accelerate the convergence of calculations, and then we have optimized the parameters for this spatial biasing technique. We have also compared our multi-parameters method BS4P to others methods with 2 parameters BS2P and the methods with 1 parameter BS1P and we have determined the FOM for each method. We have clearly identified that our method BS4P is more efficient for great thickness at high values of Ps then we have identified some advantages of our study for practical situations

In perspective, we intend to extend this study to determine the spatial spectrum of neutrons transmitted for different neutron energy spectra.

\section{REFERENCES}

[1] Booth, T. E. and Hendricks, J. S, Nuclear Technology 5, (1994).

[2] CRC Handbook of Nuclear Reactor Calculations, Roenergy. CRC Press, Vol. II, (19860, pp. 49.

[3] Khanouchi A, Sabir A, Boulkheir M, Ichaoui R, Ghassoun J, Jehouani A, Applied. Radiation and Isotopes. Vol. 48, №. 10-12 (1997): 1667-1671.

[4] David, M. C., Duct and Void in Shields, In Reactor Handbook, Vol. III, Part B, 1962, pp 166.

[5] Lee J. B, Lee W. B, Lee C. B, Nuclear Science and Engineering 121 (1995): 334-344.

[6] Khanouchi A, Fakir M, Sabri M, Jehouani A, IEEE. DOI 10.1109/CGIV 2016.96 (2016): 452-456.

[7] Khanouchi A, Fakir M, Sabri M, Jehouani A, 13th International Conference Computer Graphics, Imaging and visualization, Beni Mellal, Morocco, 29 March-1 April 2016 (2016).

[8] Jehouani A, Ghassoun J, Aboubekr A, In Proceedings of the 6th International Symposium on Radiation Physics, Rabat, Morocco, 18-22 July (1994).

[9] Levitt, L. B., Nuclear Science and Engineering 31 (1968): 500-504.

[10] Ghassoun J., doctoral thesis Faculty of Sciences Semlalia Marrakech, (1994), pp 35.

[11] Ghassoun J, Sabir A, Khanouchi A, Boulkheir M, Ichaoui R., Jehouani A, Applied. Radiation and Isotopes. Vol. 48, No. 10-12 (1997): pp. 1497-1500

[12] Khanouchi A, Fakir M, Sabri M, Jehouani A, the First Mediterranean Conference on Methanisation, Biogas and Wastes Energy Recovery, Beni Mellal, Morocco, 1-2 June 2016 